\title{
An Exposition on the Quanfeng Lantern Opera and Its Cultural Characteristics
}

\begin{abstract}
Dandan Zhou ${ }^{1, *}$
${ }^{1}$ Art Institute, Jiujiang University, Jiujiang, Jiangxi 332005, China

*Corresponding author. Email: 153523142@qq.com

ABSTRACT

Quanfeng Lantern Opera (also "Quanfeng Hua Deng", "全丰花灯"), an important local folk art form of Jiangxi Province, was included in the First National List of Intangible Cultural Heritage of China in 2006 (500IX-52). It has developed a unique performance form and characteristics after long-standing as the folk-art form closest to the people of northern Jiangxi Province. This paper analyzes and discusses all elements of the Quanfeng Lantern Opera from the perspective of art culture. It reapproaches and interprets, in a manner that may benefit the modern researches, the Quanfeng Lantern Opera based on its history and current situation, research of characteristics of all performance elements, regional characteristics and folk customs. The re-interpretation will allow us to understand the meaning behind the Quanfeng Lantern Opera, and its influence on the thoughts and behaviors of the general public. It further makes us recognize the role of the traditional cultural and artistic performances in the construction and maintenance of a harmonious socialist society. Last but not least, it sets an example for culture researchers on how to study local art.
\end{abstract}

\section{Keywords: Quanfeng Lantern Opera, performance, characteristics, culture}

\section{INTRODUCTION}

Quanfeng ${ }^{1}$ Lantern Opera ${ }^{2}$ originated from the Song Dynasty $^{3}$ of China. Just like those similar lantern-based

*Fund: The study was supported by "2017 Jiangxi University Humanities and Social Sciences Research Project — Quan Feng Hua Deng Art Research"(Grant No. YS17231).

Quanfeng Town is one of 36 towns in Xiushui County, Jiujiang City, Jiangxi Province. It was the jurisdiction of Xiping County from the Eastern Han Dynasty to the Tang Dynasty. It was renamed Xixiang, Yining County starting since 800 A.D. in the Tang Dynasty. Located in the northwest of Xiushui County, Jiujiang City, Jiangxi Province, it is a basin-shaped mountainous area surrounded by mountains, flat in the middle, adjacent to Bailing Town, Lukou Town, and Dachun Town, and bordering on Tongcheng and Chongyang counties in Hubei Province. In the area of $100.2 \mathrm{~km} 2$, there are 15 administrative villages, 243 villager groups, 7,080 households, and more than 28,000 populations. It has a warm and humid climate and is rich in natural resources. As a town with a longstanding history, it is one of the birthplaces of the Autumn Harvest Uprising.

Also known as the Lantern Opera. It is a popular opera form around China. The Lantern Opera came from lantern songs and dances of the folk. It is an opera form with local features born in the late Qing Dynasty and the early Republic of China. It has evolved into different singing and performance styles due to the influence of local dialects, folk songs, customs, etc.

The Song Dynasty (960-1279) was a dynasty sit in between the Five Dynasties and Ten Kingdoms and the Yuan Dynasty in Chinese history. It had two stages, namely the Southern Song Dynasty and the Northern Dynasty. With eighteen emperors on the throne, it lasted 319 years. arts, such as Sichuan Lantern Opera, Yun'nan Lantern Opera, Guizhou Lantern Opera and Gansu Lantern Opera, it blossomed when the Poetic Opera of the Song Dynasty ${ }^{4}$ thrived. Historical records show that the Quanfeng Lantern Opera was born when the Emperor Renzong of the Song Dynasty ${ }^{5}$ restored his power to rule from his mother Liu $\mathrm{E}^{6}$, the first regent empress dowager in Song Dynasty. Liu E came to Quanfeng

The Poetic Opera of the Song Dynasty is a general term for all kinds of singing, dancing and dramas of Song Dynasty. The Song Dynasty represents the mature period of Chinese opera. It was called "Yuan Ben (院本)" in the Jin and Yuan Dynasties. The opera started with a little piece of singing and dancing performed by five characters, called "Yan Duan (艳段)"; followed by a "main opera", usually a farce or story told against the tune of "Da Qu". The Wulin Jiushi (武林旧事) written by Zhou Mi recorded 218 official opera names.

Zhao Zhen, Emperor Renzong of the Song Dynasty (May 30, 1010-April 30, 1063), formerly as Zhao Shouyi, was the fourth emperor of Song Dynasty (March 23, 1022 - April 30, 1063 on the throne). He was the sixth son of Zhao Heng, Emperor Zhenzong of the Song Dynasty. His mother was Li Chen Fei.

Liu E (969-1033), Empress Zhangxian Mingsu, wife of Zhao Heng, Emperor Zhenzong of the Song Dynasty, was the first regent empress dowager in Song Dynasty. She has been often compared to Empress Lv of the Han Dynasty and Empress Wu of the Tang Dynasty, and was rated as "had the talents of $\mathrm{Lv}$ and $\mathrm{Wu}$ but without the evil". 
Baoshan Temple ${ }^{7}$ to practice Buddhism after giving back the power to rule, which offered an opportunity for the combination of the Royal Lantern Opera and Buddhist music. The Quanfeng Lantern Opera is a product of three Poetic Operas of the Song Dynasty, namely the "Bamboo Horse Opera ("竹马戏")" and Singing ("说唱") $)^{9 "}$ and "Royal Lantern Opera of the Song Dynasty". It inherited the contents and forms of the royal operas of the Song and Tang Dynasties, and further blended them with local dialects, music and folktales of Quanfeng to create the unique style and characteristics.

As an important cultural form, the Quanfeng Lantern Opera displays a variety of artistic forms and skills including opera, dancing, singing, dialogue, lantern-based performance, acting and acrobatic, and they further unfold as opera, dance, Quyi ("曲艺"), telling and singing, vaudeville, bamboo horse, flower drum ("地 花鼓"), folk song, lantern decoration, paper cutting, print, Bianzha ("编札"), mounting, production and light and shadow. The Quanfeng Lantern Opera is a consummate product of Chinese history and culture and the local culture of Jiangxi Province. The unique art form has always been closely related to the life of the local people and has profoundly affected the way of how people think and understand the world. The Quanfeng Lantern Opera was an important part of the local culture of northern Jiangxi Province, and even up to now, it is still the most important entertainment resort in the everyday life of the indigenous people of Quanfeng.
The Baoshan Temple was formerly the Nanfen Temple. It sits at the eastern foot of Nanfeng Mountain, Nanfeng Village, Quanfeng Town, Xiushui County. It was constructed during the year 1008-1016 when the Emperor Zhenzong of the Song Dynasty was in power and named by the emperor as "Baoshan Temple". As a very important temple in the history of Xiushui, it was included into the six major temples of Xiushui together with Huanglong Temple and Doushui Temple.

The bamboo horse opera is a local opera form developed from "Pao Zhu Ma (跑竹马)", a folk singing and dancing performance. It originated in the counties of Zhangpu and Hua'an in southern Fujian and is popular in counties (cities) such as Changtai, Nanjing, Longhai, Zhangzhou, Xiamen, Tong'an and Jinmen and Taiwan.

It is a traditional quyi form, displayed in talking or singing, such as Bianwen (变文), Pinghua (评话), Kuaishu (快书), Dagu (大鼓 ), Tanci (弹词), Daoqing (道情) and Baojuan (宝卷), etc.

\section{THE PERFORMANCE SYSTEM OF THE LANTERN OPERA (A COMBINATION OF SINGING, DANCING AND DRAMA)}

\section{A. Singing: the combination of telling and singing}

Quanfeng Lantern Opera shares some characteristics with local folk songs: simple, natural and un-carved. The singing of the Quanfeng Lantern Opera is frisk and bright, full of pleasure, just like local folk songs, despite they have been re-produced and adapted by folk artists during spreading. The tune is structured and rhythmic. The flat and low-pitch singing delivers deep feelings, mingled with passionate emotions from time to time. Basically, the single-tune has two echoing lines while the string-of-tune has two echoing qu ("曲"). The performance combines dialogue and singing, and the use of local dialect displays strong regional characteristics. The local dialect used in the telling and singing and the spoken lines of this art form is the ancient Jiangxi dialect, coming from Duchang County. (see "Fig. 1") Lots of words and expressions are inserted in almost every line of verses for balance or euphony, creating an amicable and contagious artistic atmosphere.

The features displayed in the music of the Quanfeng Lantern Opera also include: "one leading performer and multiple sing-along performers", "antiphonal singing"(see "Fig. 2"), "echoing singing by several performers" and "singing-in-unison". It combines telling and singing. The use of prolonged voice and tune creates the unique pre-tune and pro-tune displayed at the beginning and the end of each piece of Quanfeng Lantern Opera; that one performer plays several roles allows the same performer to speak or sing for himself/herself or a third person; the addition of the percussion instruments such as Chinese gong chime, gong, drum and cymbal creates a better stage atmosphere; sometimes the huqin ("胡琴"), bamboo flute and suona horn may also be used for accompaniment; all lantern operas share the same orchestral music interlude, going like De Kuang Kuang/De Kuang Kuang/De Kuang Kuang/De Kuang Kuang, and some parts of the opening, prelude and interlude share the same rhythm.

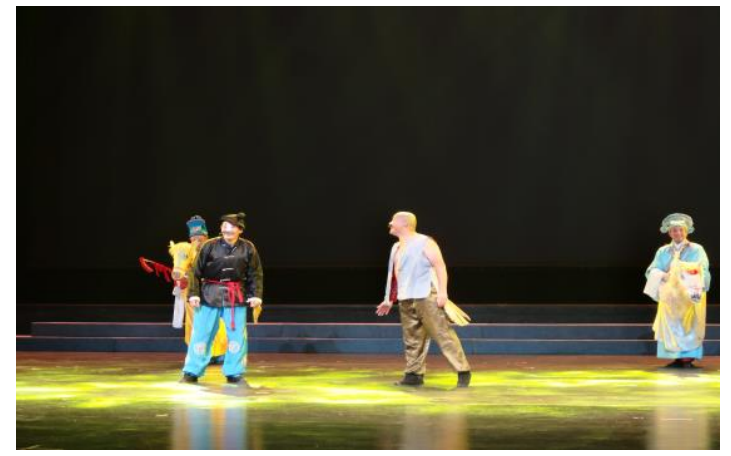


Fig. 1. Performers delivering spoken lines in a Quanfeng Lantern Opera $^{10}$.

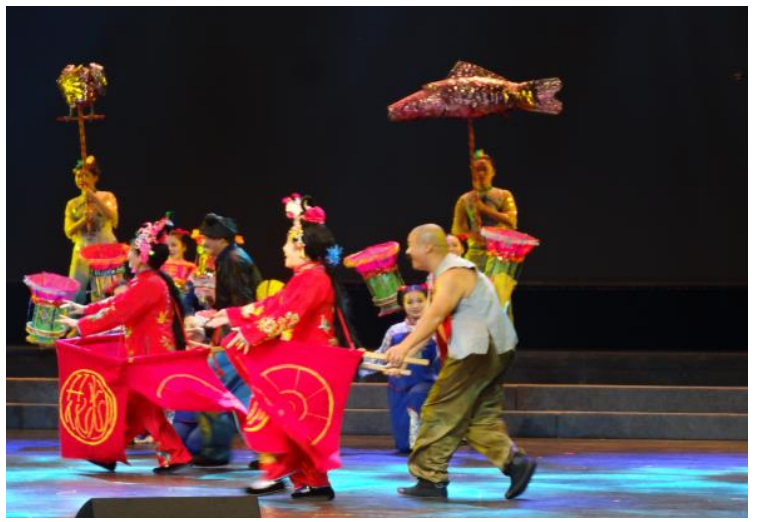

Fig. 2. Antiphonal singing in a Quanfeng Lantern Opera.

\section{B. Dancing: the combination of drama and dance}

The dance displayed in the Quanfeng Lantern Opera is combined with drama as well as ethnic and folk features. On the one hand, the body language of each character is very similar to that displayed in an opera performance. The acting displayed in the Quanfeng Lantern Opera refers to performance skills in general, especially referring to the dance-like body language. This is one of the main signs that the Quanfeng Lantern Opera is different from other performing arts. The creation of a character needs skills in the hands, eyes, body and walk; and different skills can be used in actions including whip-raising, hair-swinging, walking and appearance-making. The combination of these basic opera-performance skills and dance makes it possible to emphasize the character, age and identity of the role. The dance-like actions in those skills-oriented performances pay more attention to the revealing of the mental activities by body language, where the character creation is the ultimate goal. On the other hand, Quanfeng Town sits in the south of Jiangxi Province of China where Han people live, so the dance shares some characteristics of the folk dance of Han. It strongly reflects the life of locals and also displays pungent, funny, quiet and enthusiastic traits, such as the cross step and wrist-winding skill. It is a great tool to have the outsiders understand the simplicity, honesty and kindness of the people of Quanfeng Town.

The standing-out dance displayed in the Quanfeng Lantern Opera has effectively made this art from more welcoming and recreational. The lights used in the Quanfeng Lantern Opera has long divorced from the

10 The artists in this picture are Dai Shuiping, Dai Zhixin, Hu Caiju, Rao Meiling, Dai Dingzheng, Dai Zhifu, Yu Nanping and Yang Dahui. They are inheritors or performers of Quanfeng Lantern Opera; Art Institute of Jiujiang University; Thanks to the Ethnic and Folk Culture and Art Research Base of Jiujiang University for providing materials support. pure lighting function, and evolved to become a supplement for vocal accompaniment as well as singing and dancing. (see "Fig. 3")The use of the lights on the stage, not just the fixed-position lights, allows the performance to better suit the stage and become more professional and three-dimensional. The increase from one carriage and horse to several carriages and horses shows the improvement so it can better suit the stage.

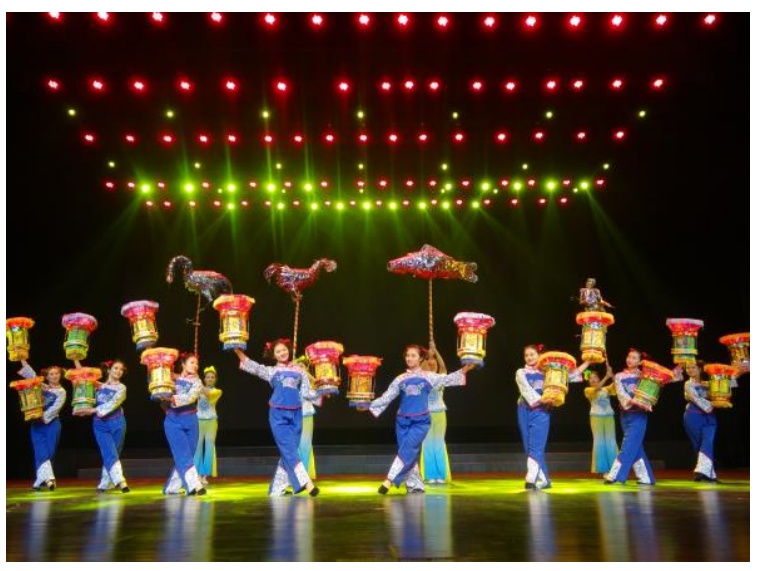

Fig. 3. Lantern dance in a Quanfeng Lantern Opera.

\section{Drama: the combination of script and improvisation}

The Quanfeng Lantern Opera requires three performers and the three is also the core of the performance: the female role (with a handkerchief in one hand and the handlebar in the other); the male role (with a hat on top and wears tofu-like facial makeup); and the clown role (who will help the carriage forward from the behind; the carriage is surrounded by curtains with wheel prints on both left and right sides), as shown in "Fig. 4" "Fig. 5". It is different from some other lantern-based performances such as the song-and-dance duet (which requires two performers), the fiveperformer opera (which requires five performers) and the seven-performer opera (which requires seven performers); instead it requires three performers. The Quanfeng Lantern Opera can be carried out with one performer playing multiple roles; and the plot of the opera is generally simple. As for the history, that the male role rides a bamboo horse originated from the "Bamboo Horse Opera" in the northwestern China of the Han and Tang Dynasties; that the female role rides a carriage inherited the "Carriage Opera ("车车戏")" in Central China; and the funny performance given by the clown role, including leg-bending while helping the carriage forward, shoulder-shaking, jumping, leapfrogging, improper dressing, belt and skullcap wearing, stemmed from the costume and makeup in the Poetic Opera of the Song Dynasty. 


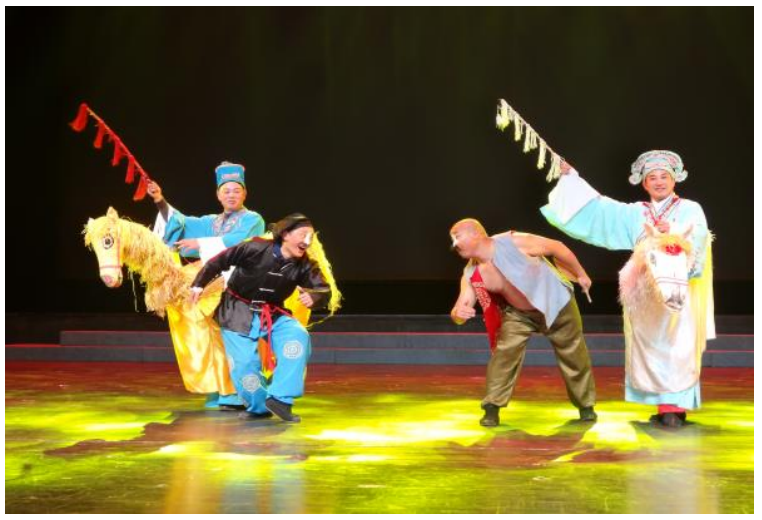

Fig. 4. A performance scene in a Quanfeng Lantern Opera (1).

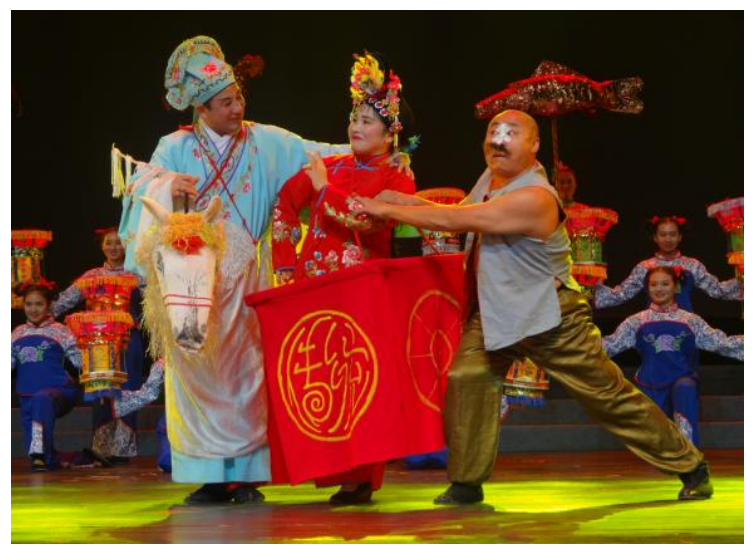

Fig. 5. A performance scene in a Quanfeng Lantern Opera (2).

\section{THE CULTURAL CONNOTATION OF THE QUANFENG LANTERN OPERA}

\section{A. Celebration of important festivals}

The Quanfeng Lantern Opera usually appears in festivals and happy occasions, especially during the Spring Festival. Sometimes the performance can last from the first day of the lunar new year to the fifteenth day, day and night. The performance groups of the town traveled from one household to another household to give performances. Colorful and bright lanterns mingled with the performance of gongs and drums light up the whole town, day and night. Not only the festivals, the lantern performance group can also be seen on occasions such as birthday-celebrating of the elderly, foundation-laying ceremony and wedding, as a way of celebration. (see "Fig. 6")
G

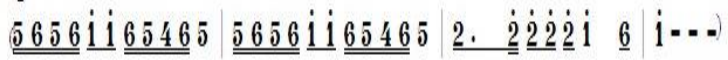

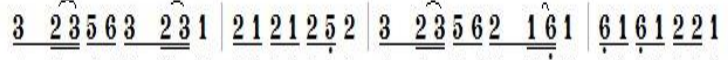

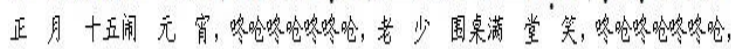

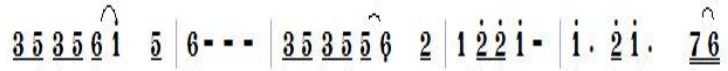
兴高采烈驺汤圆, 新昐新年福运到然咚呛。今夜星空

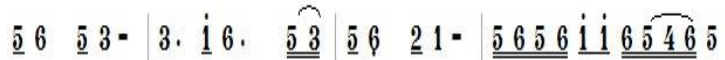

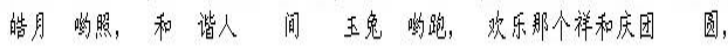

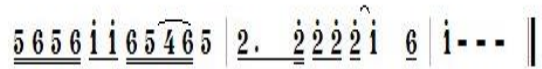
祝愿那个未来更美好，祝 愿未来更美好。

Fig. 6. The music score of the Celebration of the Lantern Festival.

\section{B. Celebration of four-season farming activities}

The music score of the Liu Ge Yue Zhong Hua is shown below. (see "Fig. 7")

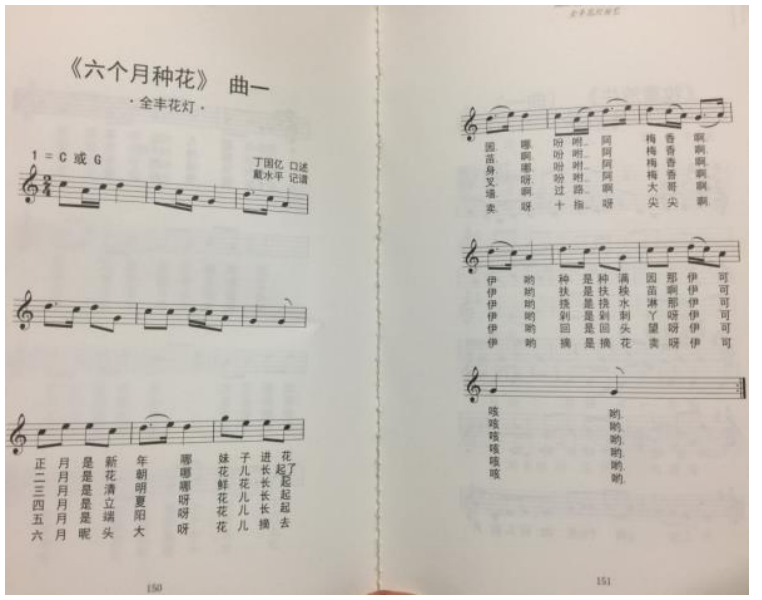

Fig. 7. The music score of the Liu Ge Yue Zhong Hua.

\section{Sacrificial activities}

Traditionally, the "lantern god-welcoming ceremony" will be held before the start of the lantern performance. The charger delivers the lantern godwelcoming letters to every household to have them prepared for the ceremony. When the ceremony starts, the lantern team carries the statue of the lantern god around in a sedan. Locals greet the god with firecrackers and worship it with incense and offerings. The lantern team will also be offered with supplies as a thank-you gesture from the locals.

\section{Creation and adaptation of new opera}

The Spring ("盛世花灯和谐春"), as a Quanfeng lantern opera, integrates three traditional lantern operas, namely the Feng Chun Hua ("逢春花"), Liu Ge Yue 
Zhong Hua ("六个月种花") and Shi Dai ("十带"). It displays an extremely-joyful scene of the Quanfeng people expecting "a peaceful and flourishing nation" and "loving the Chinese Communist Party and getting prosperity". With multiple ups and downs, the opera represents a comedy that reveals the life of the Quanfeng people. (see "Fig. 8")

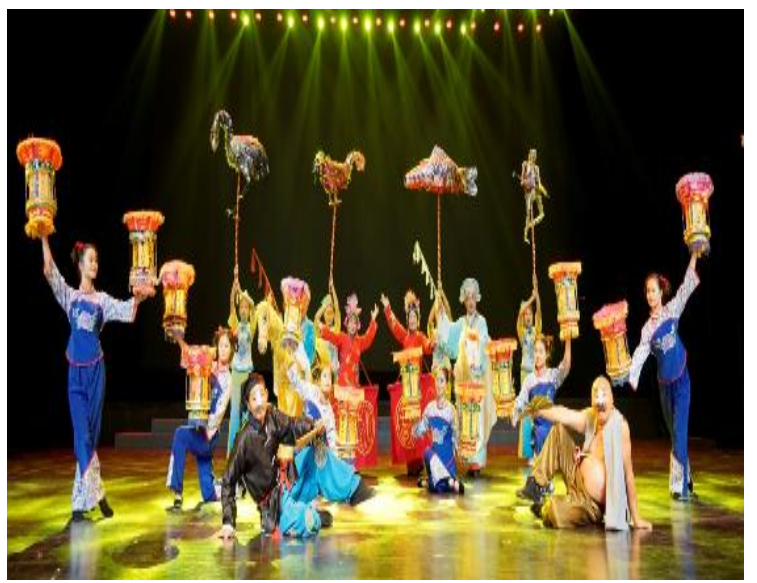

Fig. 8. A scene of the Spring performed by the Art Institute of Jiujiang University.

This piece of opera tells a story that happens on the lantern show in lunar January. It reflects a slice of life of locals: people, men and women, old and young, came to the lantern show to appreciate the lanterns, talking and laughing, singing and dancing, full of joy. It reveals the joyful and hopeful gathering of Quanfeng people at the central square to celebrate the peaceful and prosperous life they have after four-season hardworking. The "happy and promising" life displayed in the opera shares the "positive energy" upheld by the current society. This opera shows both the artistic characteristics of the traditional lantern opera and the unique local features of Quanfeng, both the real-life experience of the locals and the artistic recreation and re-production. The simplicity, sincerity and happiness displayed in this show reflect the underlying theme "prosperity and harmony" of Quanfeng.

\section{CONCLUSION}

Quanfeng Lantern Opera, as a local performance form of Jiangxi Province, is a "grassroots-based art" born in Quanfeng and full of rural and folklore features. As a popular local cultural form in Quanfeng rural area, it reflects the interaction between people and the rural society. The combination of opera and lanterns, elegance and vulgarity as well as drama and folklore in the Quanfeng Lantern Opera creates major popularity among the local people.

Quanfeng represents an important town of Jiangxi Province in terms of local culture, and the Quanfeng
Lantern Opera stands as a symbol of Quanfeng due to its high popularity. The art form is well-received for a long time both in Quanfeng and in its surrounding counties and towns. The performance organized by the locals can be seen on streets or villages as a celebration of the Spring Festival and Dragon Boat Festival every year. Not only this, the performances of dragon lanterns, lion lanterns and boat lanterns are also part of the celebration. They have contributed more joy to the festivals and allowed the town people to have a better spiritual life, simple but optimistic living conditions and endless vitality. Quanfeng Lantern Opera, as a symbol, reflects the expectations and morals of people living in this region. It is not only a reflection of the real-life experience of people living here, but also a reflection of the optimism and profound understanding of sufferings shown by ordinary people in their daily life.

\section{References}

[1] Dai Fenghong, Dai Shuiping and Yang Dahui, "Quanfeng Lantern Opera" [M] Beijing: Unity Press, 2016, pp. 34-37, 6670

[2] Yang Rui, "Research on the Regional Division of Jiangxi Folk Culture" [D] Master's thesis of Jiangxi Normal University, 2015(6) pp. 27, 33, 47

[3] Liang Chuan, "A Discussion of the Cultural Identity Characteristics of the Lantern-appreciation Convention in the Lantern Festival" [J] Folk Culture, 2014(5), P. 124

[4] Wu Xianxian, "Research on the Evolution of Intangible Cultural Heritage Based on Folklore Performances" [D] Master's thesis of Nanchang University, 2014 (5), pp. 16, 19

[5] Zhou Lin, "Quanfeng Lantern Opera: An Artistic Heritage Popular among the Locals" [J] Economic Daily, 2010 (2), p. 007

[6] Liu Man, "Research on Dances in Operas" [D] 2012 doctoral dissertation of Chinese National Academy of Arts, 2012 (5), p. 43 